F. Muratori

A. Menghi

M. Fantoni

E. Visconti

F. Pezzillo

T. Nizegorodcew

G. Maccauro

\section{Tubercular osteomyelitis of the second metatarsal bone: case report}

Received: 14 December 2006

Accepted: 20 July 2007

Published online: 21 December 2007

F. Muratori

Division of Orthopaedics

San Pietro Hospital

Rome, Italy

A. Menghi • F. Pezzillo • T. Nizegorodcew

G. Maccauro ( $\mathbb{Q})$

Department of Orthopaedics

Catholic University

Rome, Italy

E-mail: giuliomac@tiscali.it

M. Fantoni • E. Visconti

Department of Infectious Diseases

Catholic University

Rome, Italy

\begin{abstract}
Many studies have described the osteoarticular involvement of tuberculosis, but very few cases of tubercular osteomyelitis of the foot have been reported. We describe a case of spina ventosa affecting the second metatarsal bone, and review the literature to describe the clinical manifestation, imaging aspects and the treatment of skeletal tuberculosis.
\end{abstract}

Key words Spina ventosa .

Tuberculosis - Metatarsal bone .

Debridement

\section{Introduction}

Skeletal tuberculosis is relatively uncommon compared to the pulmonary form of tuberculosis. In fact, bones and joints are affected in $1 \%-3 \%$ of all cases $[1,2]$, and the spine and the hip are most commonly involved. Localization in the foot is very rare, and accounts for only about $10 \%$ of all cases of skeletal tuberculosis. We describe the clinical manifestation, imaging aspects and treatment of a case of skeletal tuberculosis of the second metatarsal bone, not previously described in the literature.

\section{Case report}

A 29-year-old man presented with a 2-month history of pain, swelling and ulcer of the dorsal region of the left foot, and was admitted to our department. Radiographs showed a cystic, expansive lesion of the second metatarsal bone (Fig. 1a) with internal septations and cortical sclerosis. The margins were well defined, and periosteal reaction was present. Computed tomography (CT) showed an osteolytic area involving the entire second metatarsal bone, with an expansive mass above the bone that invad- 

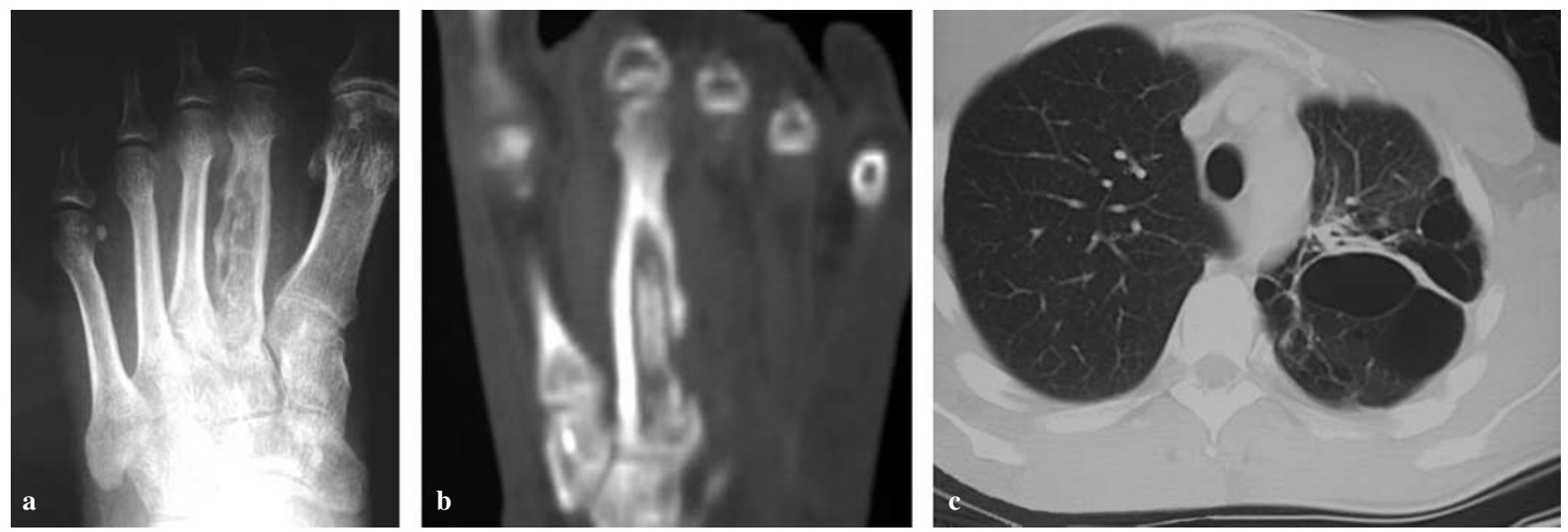

Fig. 1 a-c Tubercular osteomyelitis of the second metatarsal bone. a Radiograph shows a cystic, expansive lesion of the second metatarsal bone. Bone trabeculae are visible inside the canal. Lateral cortex appears thinner, in comparison to the medial one. b CT scan shows an osteolytic area involving the whole second metatarsal bone with an expansive mass above the bone that has invaded the second metatarsal space. Bone sequestrum is visible inside the medullary canal. c CT scan of the chest shows a pulmonary cavernous lesion surrounded by fibrosis

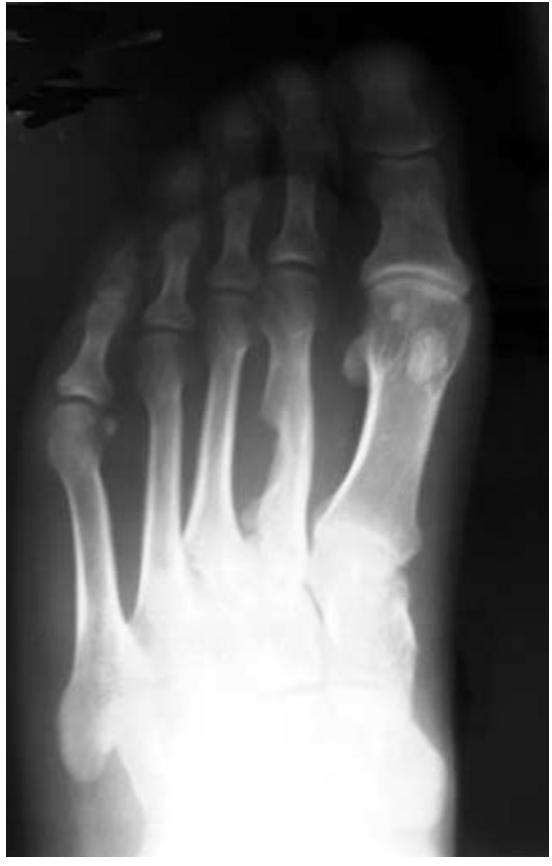

Fig. 2 Radiograph showing absence of recurrence of the bone lesion

ed the second metatarsal space. Bone sequestra were visible inside the metatarsal bone (Fig. 1b). Magnetic resonance imaging (MRI) showed a signal alteration of the second metatarsal bone, with a large soft mass surrounding the lesion, in particular over the second metatarsal bone and in the metatarsal space. CT of the chest showed a pulmonary lesion (Fig. 1c). Haematological and chemical parameters were normal and the erythrocyte sedimentation rate $(\mathrm{ESR})$ was $19 \mathrm{~mm} / \mathrm{h}$.
A fine-needle biopsy previously performed in a different hospital had excluded neoplasm and suggested a diagnosis of osteomyelitis. We performed an open biopsy, with debridement and curettage of the lesion. Histological examination of the tissue showed acid fast bacilli with granulomatous inflammation. A diagnosis of Mycobacterium tuberculosis infection was made on the basis of culture and genome amplification by PCR. The patient was from a country where the mycobacterial infection is endemic.

Antitubercular treatment was started with rifampin $(600$ $\mathrm{mg}$ /day), isoniazide (300 $\mathrm{mg} /$ day), ethambutol (1200 $\mathrm{mg} /$ day), and pyrazinamide (2000 mg/day). Three weeks later, when the antibiogram was available, ethambutol was discontinued; after eight weeks pyrazinamide was also discontinued. Rifampin and isoniazide were continued for a total of 52 weeks. After two weeks of therapy, the cutaneous ulcer healed completely, and after four weeks radiographs showed that the lesion had healed. At the 6-month follow-up, the patient did not show any local or systemic recurrence (Fig. 2).

\section{Discussion}

The most commonly affected sites of tuberculous osteomyelitis are the spine, femur, tibia and fibula. Less frequently affected is the hand [3-5]. Skeletal tuberculosis of the foot is very uncommon and usually affects the calcaneus, talus, first metatarsal bone, navicular bone and medial and intermediate cuneiform bones [1, 2, 6].

The diagnosis depends on clinical and radiological aspects, laboratory tests, and microbiological and histological features. The clinical manifestation consists of 
pain, swelling, stiffness and muscle atrophy. A chronic discharging sinus or chronic skin ulcer may be present [7]. ESR is normally elevated, but a normal value and a negative skin test cannot exclude a diagnosis of tuberculosis.

The radiological pattern is usually osteoporosis, bone expansion with reactive sclerosis, periosteal reaction, reduced joint space, soft-tissue swelling and progressive destruction of the joint, with a typical aspect of cystic expansion of the short tubular bones named "spina ventosa" [1-3, 8-10]. MRI is the first-choice technique to reveal the soft-tissue, and marrow involvement, before plain radiography. Biopsy is indicated for the differential diagnosis of several bone diseases.

A long-term course of antitubercular drugs is the basis of the treatment. Some authors suggested a minimum treatment of 12 months for osteoarticular involvement [6-8]. Debridement and curettage without resection of destroyed or sequestrated metatarsal bone may be indicated in non-healing lesions. In the present case, the large involvement of soft tissue suggested a need for debridement and curettage, followed by an antitubercular treatment, usually rifampin, isoniazide, ethambutol and pyrazinamide. Rifampin and isoniazide were continued for 52 weeks while ethambutol and pyrazinamide were discontinued after the antibiogram was available. Good functional results have been achieved with conservative treatment in cases affecting the metatarsal and phalanges, and rehabilitation has been successful even when joint destruction was present [3]. Resection of the destroyed metatarsal bone is rarely necessary.

\section{References}

1. Mittal R, Gupta V, Rastogi S (1999) Tuberculosis of the foot. J Bone Joint Surg Br 81(6):997-1000

2. Dhillon MS, Sharma S, Gill SS, Nagi ON (1993) Tuberculosis of bones and joints of the foot: an analysis of 22 cases. Foot Ankle 14:505-513

3. Subasi M, Bukte Y, Kapukaya A, Gurkan F (2004) Tuberculosis of the metacarpals and phalanges of the hand. Ann Plastic Surg 53(5):469-472
4. Andronikou S, Smith B (2002) Spina ventosa-tuberculous dactylitis. Arch Dis Child 86(3):206

5. Benkeddache Y, Gottesman H (1982) Skeletal tuberculosis of the wrist and hand: a study of 2 cases. J Hand Surg 7(6):593-600

6. Dhillon MS, Nagi ON (2002) Tuberculosis of the foot and ankle. Clin Orthop Relat Res (398):107-113

7. Yuen MC, Tung WK (2001) An uncommon cause of foot ulcer: tuberculosis osteomyelitis. Emerg Med J 18(2):140-114
8. Vervest TM, Nollen AJ, de Munck DR (1998) A case of spina ventosa. Acta Orthop Scand 69(3):322-323

9. Watts HG, Lifeso RM (1996) Current concepts review: tuberculosis of bone and joints. J Bone Joint Surg Am 78:288-298

10. Versfeld GA, Solomon A (1982) A diagnostic approach to tuberculosis of bones and joints. J Bone Joint Surg $\mathrm{Br}$ 64:446-449 